\title{
Diversidad de musgos en comunidades vegetales asociadas a una pingüinera en la Isla Decepción, Antártica marítima
}

\section{Moss diversity in plant communities associated with a penguin rookery on Deception Island, Maritime Antarctica}

\section{Cristobal Araneda ${ }^{1}$, José María Fernández ${ }^{2}$, Marc Oliva ${ }^{2,3}$, Götz Palfner ${ }^{1}$ \& Angélica Casanova-Katny ${ }^{4, *}$}

${ }^{1}$ Laboratorio de Micología y Micorrizas, Departamento de Botánica, Facultad de Cs. Naturales y Oceanográficas, Universidad de Concepción, Barrio Universitario s/n, Concepción, Chile.

${ }^{2}$ Instituto de Geografía e Ordenamento do Território (IGOT), Universidade de Lisboa, Rúa Branca de Edmée Marques, Cidade Universitária, Lisboa, Portugal.

${ }^{3}$ Departamento de Geografía, Facultad de Geografía e Historia, Universitat de Barcelona, Montalegre 6, Barcelona, España.

${ }^{4}$ Laboratorio de Ecofisiología Vegetal y Cambio Climático y Núcleo de Estudios Ambientales (NEA), Facultad de Recursos Naturales, Universidad Católica de Temuco, Rudecindo Ortega 03694, Temuco, Chile.

*E-mail: mcasanova@uct.cl

\section{RESUMEN}

Con el objetivo de determinar la influencia de las pingüineras sobre la diversidad de la vegetación en la Isla Decepción, se estudió la composición de briófitas de un transecto de aproximadamente $2 \mathrm{~km}$ entre el Lago Irízar y la pingüinera (Pygoscelis antarcticus) de Punta La Descubierta. Fueron detectados un total de 39 carpetas de vegetación formadas principalmente por briófitos, distribuidas en tres sectores principales, aledaño al Lago Irízar, en Collado Vapor y en Punta La Descubierta. Los briófitos registrados corresponden a 15 especies de musgos y sólo 2 hepáticas, con 11 familias representadas. Se detectó que dos musgos, Sanionia uncinata y Politrychastrum alpinum, con 31 y 9 registros, dominaban las carpetas, con mayor abundancia y frecuencia. Además, se encontró un nuevo registro para la isla del musgo Bryum orbiculatifolium, el cual crece directamente asociado a la pingüinera. Además, se encontró que hay 5 especies de musgos comunes a los tres sectores, aunque otras 5 especies crecen solo en el sector de la pingüinera, diferenciándose esta comunidad de las otras dos. No se detectaron plantas vasculares en todo el sitio de estudio, por lo que se discute la posibilidad de que estos sitios se encuentran en estados de colonización temprana y donde su biota está marcada por la presencia de musgos pioneros que crecen alrededor de las pingüineras, influenciadas probablemente por el aporte de nutrientes del guano depositado.

Palabras clave: tundra polar, ecosistema terrestre, Península Antártica, pingüinos.

\section{ABSTRACT}

In order to determine the influence of a penguin rookery on the diversity of the vegetation on Deception Island, Maritime Antarctica, the composition of bryophytes along a transect of approximately $2 \mathrm{~km}$ between Lake Irízar and the penguin rookery (Pygoscelis antarcticus) of La Descubierta Point was studied. A total of 39 vegetation carpets formed mainly by bryophytes were detected, distributed between three main sectors, next to Lake Irízar, in Vapour Coil and at La Descubierta Point. The bryophytes correspond to 15 moss species and only 2 liverworts, with 11 moss families represented. Two mosses, Sanionia uncinata (31 records) and Polytrichastrum alpinum (9 records), were found to dominate the moss carpets, being 
also the most frequent species. In addition, a new site on the island was found with presence of the moss Bryum orbiculatifolium, which grows directly associated with the penguin rookery. Our results also show that there are 5 species of mosses common to the three sectors, another 5 species grow only in the penguin area, differentiating this community from the other two sectors. Vascular plants were not detected, indicating that the studied sites are in state of early colonization where their biota is marked by the presence of pioneer mosses associated to the penguin colony, which probably benefit from the nutrient input derived from the deposited guano.

Keywords: polar tundra, terrestrial ecosystem, Antarctic Peninsula, penguins.

\section{INTRODUCCIÓN}

El continente Antártico, el quinto de mayor extensión y más austral de todos, es un territorio de clima polar, donde las condiciones son inhóspitas para la mayoría de las especies terrestres. Sin embargo, esto no es un impedimento para el establecimiento de la tundra polar, conformada en su mayoría por especies no vasculares (Convey 2005, Casanova-Katny \& Cavieres 2012). Esta flora antártica está restringida a zonas costeras libres de hielo (Convey et al. 2014), por lo que su distribución es parchada y determinada por la disponibilidad local de zonas libres de hielo glaciar y con agua biodisponible (Robinson et al. 2018). Este tipo de hábitat se encuentra con relativa frecuencia a lo largo de la zona oeste de la Península Antártica, donde además las condiciones climáticas húmedas y frías, pero con temperaturas medias mensuales positivas durante el verano, favorecen la colonización, principalmente por especies criptogámicas, entre ellas se han registrado alrededor de 350 especies de líquenes, 100 musgos, 25 hepáticas (Øvstedal \& Lewis Smith 2001, Convey 2005), y dos especies de plantas vasculares (Casanova-Katny \& Cavieres 2012). En Isla Decepción, de origen volcánico, los estudios de la diversidad y desarrollo de la vegetación se han centrado en zonas de actividad geotérmica, donde la combinación de calor, vapor, inestabilidad del sustrato y concentraciones de nutrientes a menudo insignificantes o con niveles tóxicos de gases sulfurosos y depósitos minerales, imponen severas restricciones a la biota vegetal y liquénica (Smith 2005). A su vez, estos sectores ligados a un activo vulcanismo y elevada actividad geotérmica pueden haber jugado un papel determinante en la colonización vegetal del continente antártico (Convey et al. 2020), por lo que una caracterización precisa y detallada de la distribución de especies vegetales puede ayudar en una mejor comprensión de los patrones espacio-temporales de expansión de la vegetación de la Antártica. Desde el punto de vista botánico, Isla Decepción es particularmente importante, pues alberga un gran número de especies poco comunes de la Antártica, entre ellas musgos, hepáticas, y líquenes (Smith 2005). Esto ha contribuido a que, según este autor en 2005, se declaren 11 subsitios en la isla que corresponden a la Zona Antártica Especialmente Protegida, ZAEP $N^{\circ} 140$, sitios que se ubican principalmente al interior de la isla, en zonas geotérmicas o en zonas que no han sido afectadas por las erupciones volcánicas. Sin embargo, y dado que se ha descrito que las pingüineras en la Antártica influencian los patrones de distribución de la vegetación, porque aportan nutrientes (Smykla et al. 2007, Bokhorst et al. 2019), se realizó un estudio de las comunidades de musgos en la Isla Decepción en zonas sin actividad geotérmica y colindantes a una de las dos grandes colonias de pingüinos, para determinar la composición de estas comunidades vegetales y cómo son influenciadas por esta colonia.

\section{MATERIALES Y MÉTODOS}

ÁREA DE EsTUdıO

Se trabajó en la Isla Decepción ( $62^{\circ} 57^{\prime} \mathrm{S} 60^{\circ} 38^{\prime} \mathrm{W}$ ) que forma parte del Archipiélago de las Islas Shetland del Sur, específicamente, en el área comprendida entre el Lago Irízar y la pingüinera de Bahía Descubierta, el cual es accesible mediante un sendero a pie (Fig. 1).

La Isla Decepción corresponde a una isla volcánica con su caldera inundada. Las rocas más antiguas (pre-caldera) son las del grupo Puerto Foster, con alrededor de 750 ka de antigüedad (Smellie 2002). Según los registros existentes, han ocurrido 4 erupciones volcánicas en la isla en los últimos 200 años: 1842, 1967, 1969, y 1970 (Pallàs et al. 2001), por lo que gran parte de la isla se encuentra cubierta de material piroclástico. Los suelos son poco profundos y con frecuencia presentan horizontes líticos o paralíticos, con un alto contenido de gravas (30-90\%) y una textura arenosoesquelética (Bockheim 2015).

En cuanto a las condiciones climáticas, la temperatura media anual del aire en la Isla Decepción es cercana a $-2{ }^{\circ} \mathrm{C}$ al nivel del mar (Simonov 1977). La temperatura media de los 
meses más cálidos (enero) y más fríos (agosto) es $1.1^{\circ} \mathrm{C}$ y $-10^{\circ} \mathrm{C}$, respectivamente. La precipitación media anual es de $510 \mathrm{~mm}$, de los cuales $100 \mathrm{~mm}$ caen como lluvia durante el verano. El promedio de cobertura de nubes es del $50 \%$, y la humedad relativa generalmente oscila entre el 80 y el $90 \%$ (Bockheim 2015).

La isla es la menos glaciada del archipiélago, con hasta un $60 \%$ de su superficie cubierta por el hielo (López-Martínez et al. 2012). Las condiciones climáticas determinan un ambiente libre de hielo dominado por la actividad periglaciar con presencia de suelo permafrost hasta el nivel del mar. La alta porosidad del terreno volcánico condiciona una capa activa del permafrost muy superficial, situándose generalmente entre 30 y $50 \mathrm{~cm}$ de profundidad (De Pablo et al. 2017; Goyanes et al. 2014; Ramos et al. 2017). La poca cobertura vegetal favorece la actividad geomorfológica, con una dinámica periglaciar de laderas muy activa (solifluxión, deslizamientos, etc.), lo que a su vez impide el establecimiento de la cobertura vegetal.
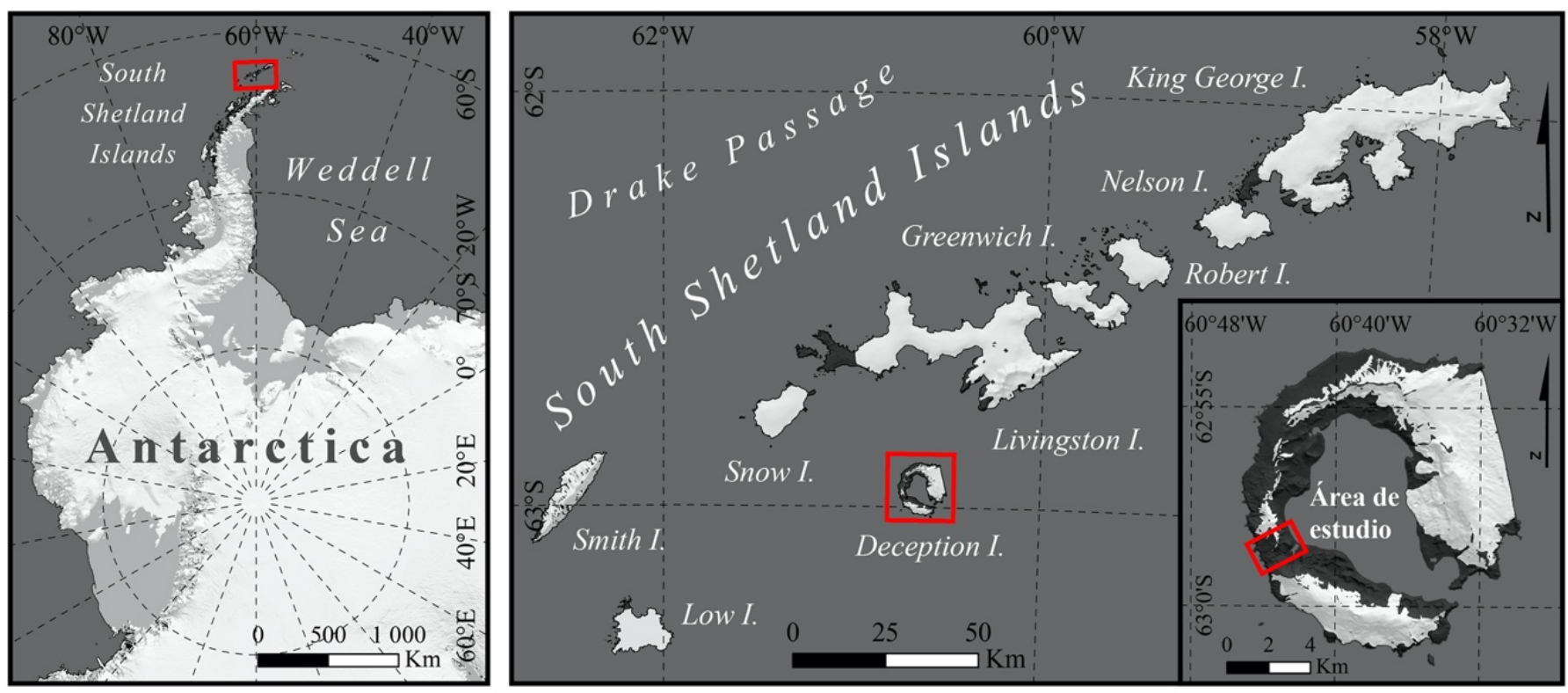

Figura 1: Ubicación de la Isla Decepción en el Archipiélago de las Shetland del Sur, en la Península Antártica. En el recuadro rojo se resalta el área de trabajo específica de este estudio. / Location of Deception Island in the South Shetland Islands, Antarctic Peninsula. The specific work area of this study is highlighted in the red box.

Metodología de Trabajo

Se definió un transecto desde el Lago Irízar, ubicado en el interior de la caldera de la isla, hasta la pingüinera de Punta Descubierta, ubicada en el exteriory orientada hacia el estrecho de Bransfield, con la finalidad de determinar variaciones en la composición de especies desde áreas altamente influenciadas por colonias de pingüinos, hacia zonas con escasa o nula influencia. Para determinar la influencia de las pingüineras, se definieron tres sectores, cada uno con múltiples sitios de muestreo, en función de la cantidad de parches de vegetación detectados. Se registró la variación en la composición y abundancia de las briófitas en un transecto que incluye tres sectores. Un sector aledaño a la pingüinera (sector de Punta La Descubierta), un sector intermedio (Collado Vapor) y un sector alejado de la Pingüinera (área alrededor del Lago Irízar).
En cada sector se recolectaron muestras de vegetación, considerando todas las especies avistadas entre los distintos parches. Las muestras recolectadas fueron secadas en un deshidratador portátil a $60^{\circ} \mathrm{C}$ por 48 horas, y posteriormente se etiquetaron y herbarizaron para su identificación. Luego en Chile, fueron enviadas al Instituto de Biología de la Universidad Católica de Valparaíso, donde se identificaron e incorporaron al herbario del briólogo Dr. Juan Larraín Benoit. Una vez identificadas, se comparó la composición de especies encontradas en cada sector mediante el índice de similitud de Sørensen-Dice (Sørensen 1957). La distribución global de las especies detectadas fue extraída de Ochyra et al. (2008), y consiste en las siguientes categorías: a) Endémica Antártica (especie exclusiva del continente Antártico), b) Subantártica (especie que se distribuye principalmente desde latitudes 
subantárticas hacia la zona norte de la península Antártica), c) Anfiatlántica (especie distribuida en ambos extremos del océano Atlántico habitando localidades en la Antártica Este y Oeste), d) Circumpolar (especie presente en localidades a lo largo del círculo polar antártico), e) Bipolar sin presencia en los trópicos (especie distribuida únicamente en altas latitudes, en ambos hemisferios; f) Bipolar con presencia en los trópicos (especie distribuida en ambos hemisferios, presente también en zonas de alta montaña en latitudes tropicales), g) Cosmopolita (especie con un amplio rango ecológico y gran distribución, algunas de estas pueden incluso habitar en zonas templadas). Se utilizó el software Primer6 para el Análisis de Similitud de Bray-Curtis para establecer los agrupamientos de las especies en los distintos sectores.

\section{RESULTADOS}

Se encontró un total de 39 parches de vegetación distribuidos a lo largo del transecto. Estos estaban dominados por briófitos, sin presencia de plantas vasculares antárticas. En total se detectaron 15 especies de musgos y 2 de hepáticas, distribuidas de forma irregular en todos los sitios de muestreo (Tabla 1). Las especies pertenecen a 11 familias, incluyendo 9 familias de briófitas (Amblystegiaceae,
Bartramiaceae, Brachytheciaceae, Bryaceae, Ditrichaceae, Grimmiaceae, Mniaceae, Polytrichaceae, Pottiaceae) y dos familias de hepáticas (Cephaloziellaceae, Jungermanniaceae). Los musgos más frecuentes fueron Sanionia uncinata (Hedw.) Loeske (31 registros) y Polytrichastrum alpinum (Hedw.) G.L.Sm. (9), aunque claramente en extensión y cobertura domina Sanionia uncinata a lo largo de los tres sectores. Por otra parte, desde el punto de vista biogeográfico, la mayor parte de las briófitas (6) presentan una distribución bipolar con presencia en los trópicos incluyendo a las dos hepáticas (Bryum pseudotriquetrum (Hedw.) G.Gaertn., B.Mey. \& Scherb., Pohlia cruda (Hedw.) Lindb., Polytrichastrum alpinum, Sanionia uncinata, Cephaloziella varians (Gottsche) Steph., Lophozia excisa (Dicks.) Dumort.), 3 especies tienen distribución subantártica y anfiatlántica (Syntrichia filaris (Müll.Hal.) R.H.Zander, Syntrichia magellanica (Mont.) R.H.Zander, Syntrichia saxicola (Cardot) R.H.Zander), y le siguen con 2 especies las de distribución anfiatlántica (Bartramia patens Brid., Bryum orbiculatifolium Cardot \& Broth.), cosmopolita (Bryum argenteum Hedw., Ceratodon purpureus (Hedw.) Brid.), bipolar sin presencia en los trópicos (Hennediella heimii (Hedw.) R.H.Zander, Sanionia georgicouncinata (Müll.Hal.) Ochyra \& Hedenäs). Además, una sola especie es endémica de Antártica (Schistidium antarctici (Cardot) L.I.Savicz \& Smirnova) y una circumpolar subantártica (Brachythecium austrosalebrosum (Müll.Hal.) Kindb.) como se muestra en la Tabla 1.

TABLA 1: Lista de especies de briófitas registradas a lo largo del transecto desde el lago Irízar hacia la pingüinera de Punta Descubierta, su familia, distribución global (extraído y modificado de Ochyra et al. 2008), número total y sector de registros en este estudio. Familias marcadas con un $\left(^{*}\right)$ corresponden a especies hepáticas / List of bryophyte species, family, global distribution (extracted and modified from Ochyra et al. 2008), number and sector of register along the transect. Families marked with $\left({ }^{*}\right)$ corresponds to liverworts species.

\begin{tabular}{lll}
\hline Familia & Especie & Distribución global \\
\hline Musgos & & \\
\hline Amblystegiaceae & $\begin{array}{l}\text { Sanionia georgicouncinata (Müll.Hal.) Ochyra \& } \\
\text { Hedenäs }\end{array}$ & Bipolar, sin presencia en los trópicos \\
& Sanionia uncinata (Hedw.) Loeske & Bipolar, con presencia en los trópicos \\
\hline Bartramiaceae & Bartramia patens Brid. & Anfiatlántica \\
\hline Brachytheciaceae & Brachythecium austrosalebrosum (Müll.Hal.) Kindb. & Subantártica circumpolar \\
\hline
\end{tabular}




\begin{tabular}{|c|c|c|c|}
\hline Familia & Especie & Distribución global & $\begin{array}{l}\mathrm{N}^{\circ} \text { de } \\
\text { registros }\end{array}$ \\
\hline \multirow[t]{3}{*}{ Bryaceae } & Bryum argenteum Hedw. & Cosmopolita & 1 \\
\hline & Bryum orbiculatifolium* Cardot \& Broth. & Anfiatlántica & 1 \\
\hline & $\begin{array}{l}\text { Bryum pseudotriquetrum (Hedw.) G.Gaertn., B.Mey. } \\
\text { \& Scherb. }\end{array}$ & Bipolar, con presencia en los trópicos & 7 \\
\hline Ditrichaceae & Ceratodon purpureus (Hedw.) Brid. & Cosmopolita & 2 \\
\hline Grimmiaceae & Schistidium antarctici (Cardot) L.I.Savicz \& Smirnova & Endémica Antártica & 2 \\
\hline Mniaceae & Pohlia cruda (Hedw.) Lindb. & Bipolar, con presencia en los trópicos & 1 \\
\hline Polytrichaceae & Polytrichastrum alpinum (Hedw.) G.L.Sm. & Bipolar, con presencia en los trópicos & 9 \\
\hline \multirow[t]{4}{*}{ Pottiaceae } & Hennediella heimii (Hedw.) R.H.Zander & Bipolar, sin presencia en los trópicos & 1 \\
\hline & Syntrichia filaris (Müll.Hal.) R.H.Zander & Subantártica, anfiatlántica & 5 \\
\hline & Syntrichia magellanica (Mont.) R.H.Zander & Subantártica, anfiatlántica & 2 \\
\hline & Syntrichia saxicola (Cardot) R.H.Zander & Subantártica, anfiatlántica & 3 \\
\hline \multicolumn{4}{|l|}{ Hepáticas } \\
\hline Cephaloziellaceae & Cephaloziella varians (Gottsche) Steph. & Bipolar, con presencia en los trópicos & 3 \\
\hline Jungermanniaceae & Lophozia excisa (Dicks.) Dumort. & Bipolar, con presencia en los trópicos & 1 \\
\hline
\end{tabular}

El índice de Sorensen, incluyendo tanto las especies de musgos como hepáticas, entre los sitios de Punta Descubierta y Collado Vapor resultó en una similitud de 66,67\%, compartiendo 8 especies de un total de 16 detectadas en ambos sitios. Entre los sitios de Punta Descubierta e Irízar, el índice fue igual a 50\%. Finalmente, entre los sitios Collado Vapor e Irízar, el índice mostró un valor de 66,7\%. Un total de 17 especies fueron registradas a lo largo de toda la transecta que cubría los tres sectores. De éstas, 5 fueron comunes a los tres sectores (Bartramia patens, Bryum pseudotriquetrum, Cephaloziella varians, Polytrichastrum alpinum, y Sanionia uncinata) de acuerdo a la Fig. 2. Otras 4 especies fueron registradas en sólo dos de los tres sectores, como se muestra en la Fig. 2. Por otro lado 8 especies fueron exclusivas de uno de los tres sectores, donde 5 de ellas fueron encontradas particularmente en el sector de Punta La Descubierta, aledaña a la pingüinera (Bryum argenteum, Bryum orbiculatifolium, Hennediella heimii, Ceratodon purpureus, Lophozia excisa). 


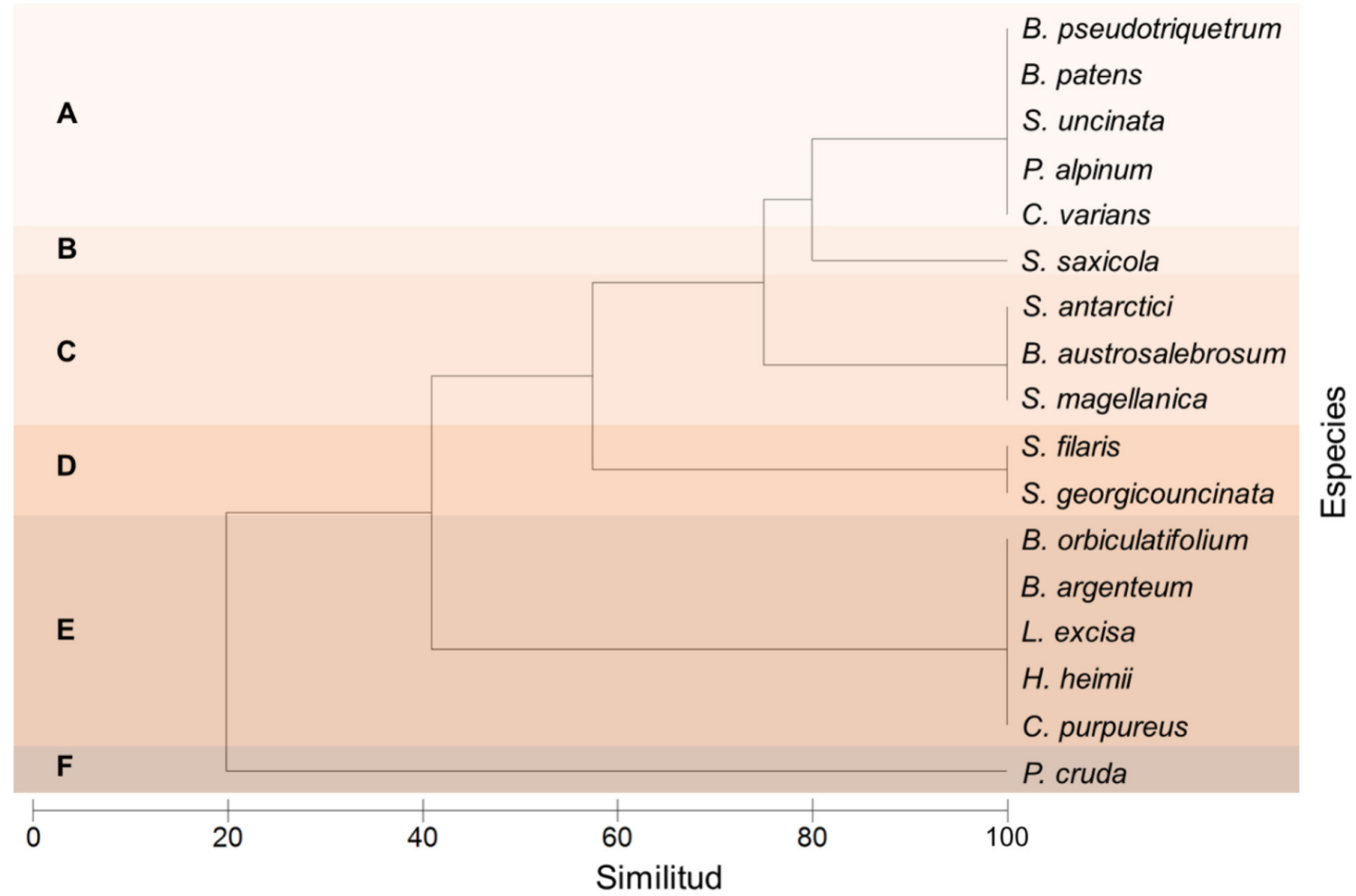

Figura 2: Análisis de Similitud de Bray-Curtis entre especies, comparando su presencia/ausencia entre sectores. Las especies resultaron agrupadas según los sitios en los que estuvieron presentes, como se denota a la izquierda con las letras $A$ a la $F$. A= especies en los tres sectores; $\mathrm{B}$ = especies en Collado Vapor e Irízar; $\mathrm{C}=$ especies en Collado Vapor y Punta La Descubierta; $\mathrm{D}=$ exclusivas de Collado Vapor; $\mathrm{E}=$ Exclusivas de Punta La Descubierta; $\mathrm{F}=$ exclusivas de sector Irízar. / Bray-Curtis similarity analysis between species, comparing their presence/absence between sectors. Species were grouped according to the site where they were present, as indicated on the left of the figure with letters A to F. A = species in the three sectors; B = species in Vapor Col and Irízar; C = species in Vapor Col and La Descubierta point; $\mathrm{D}=$ exclusive to Vapor Col; $\mathrm{E}$ = exclusive to La Descubierta point; $\mathrm{F}$ = exclusive to Irízar sector.

\section{DISCUSIÓN}

En isla Decepción prácticamente toda la vegetación descrita ha sido asociada a sitios con actividad geotérmica (Smith 2005) y no hay registros previos de flora en los sectores explorados en este trabajo. Sin embargo, nuestros resultados muestran que efectivamente existe un desarrollo de vegetación en la zona cerca de la pingüinera y sitios adyacentes, como ha sido descrito para otros sitios en las islas Shetland del Sur como el Bahía Almirantazgo (Smykla et al. 2007), o en Península Byers en Isla Livingstone y en isla Signy (Bokhorst et al, 2019). Composicionalmente, el índice de Sorensen muestra una diferenciación del 33\% entre Punta la Descubierta y Collado Vapor, y un $50 \%$ de disimilitud entre Punta la Descubierta e Irizar. Además, diferencias significativas fueron detectadas tanto en la cantidad de parches vegetacionales, como en el área promedio y total de éstos en cada uno de los sectores, siendo Punta la Descubierta el sitio más poblado de vegetación, y el más rico en especies. En este sentido, la colonia de pingüinos podría ser la principal explicación a dichas diferencias, debido a la enorme cantidad de nutrientes suministrados por los pingüinos, los cuales pueden depositar toneladas de guano en el sustrato durante las épocas de apareamiento (Smykla et al. 2007), convirtiéndose en bombas de nutrientes (principalmente $\mathrm{N}$ y $\mathrm{P}$ ) entre el mar y la tierra (Otero et al. 2018), los cuales pueden volatilizarse y dispersarse a través del viento, depositándose nuevamente en el suelo y sobre los musgos, algunos cientos de metros lejos de la pingüinera (Bockhorst et al. 2019). De esta forma, se sugiere que la fertilización causada por los desechos de los pingüinos estaría favoreciendo el establecimiento de una mayor cantidad de vegetación, debido a la mayor cantidad de 
nutrientes disponibles en comparación a las zonas interiores, donde la influencia de estas aves se ve disminuida a causa de la usualmente rápida deposición del $\mathrm{N}$ volatilizado (Skinner et al. 2006).

Aun así, la presencia de nutrientes no es el único factor determinante en la distribución de las especies; se observó una ausencia total de musgos en las zonas inmersas entre las colonias de pingüinos, y hasta alrededor de $100 \mathrm{~m}$ a la redonda en algunos casos. Dicho fenómeno ha sido descrito anteriormente, y atribuido a las nocivas cantidades de $\mathrm{N}$ derivados del guano (Tatur \& Myrcha 1989, Tatur 2002), $y$ al incesante pisoteo de los pingüinos al andar (Smykla et al. 2007), lo que imposibilita el establecimiento de musgos y hepáticas, más no de algas terrestres (e.g. Prasciola crispa) (Tatur \& Myrcha 1989).

Desde el punto de vista florístico, aunque 5 de las 17 especies se comparten entre los distintos sectores, cabe señalar que el sector influenciado por la pingüinera de Punta La Descubierta, se caracteriza además por presentar 5 especies incluyendo una hepática que sólo crecen en esa área, sector que marca una diferencia notable con los otros dos en Irízar y Collado Vapor. Además, desde Punta La Descubierta hasta el Lago Irízar, las especies con una mayor cobertura corresponden a P. alpinum y S. uncinata, quedando el resto de las especies relegadas a pequeños espacios entre los cojines y carpetas formados por ambas especies dominantes. Si bien ya se ha descrito que estas dos especies pueden desarrollarse generando carpetas de varias hectáreas de extensión, conviviendo a la vez con otras especies (Ochyra et al. 2008) y facilitando el establecimiento de plantas vasculares (Casanova-Katny \& Cavieres 2012), poco se ha mencionado sobre la notoria dominancia local de esta de $S$. uncinata especie en un área de estudio determinado. Coincidentemente, Victoria et al. (2013), al realizar los primeros mapeos de vegetación en Hennequin Point (Isla Rey Jorge) observó una significativamente mayor extensión de los parches de $S$. uncinata en comparación al resto de las especies de musgos, remarcando las características de colonizador temprano de este musgo bipolar, que también ocurre en la tundra polar ártica.

Desde el punto de vista de la distribución, la comunidad está constituida principalmente por especies ampliamente distribuidas, dado que las 15 especies de musgos ya habían sido registradas en la Isla Decepción, siendo 14 de ellas catalogadas como frecuentes para la Antártica. Además, 13 de ellas poseen una distribución amplia en la península, existiendo registros en variadas latitudes e islas. Solo se encontró una especie antártica endémica (Schistidium antarctici) y Syntrichia saxicola que posee una distribución antártica restringida a la zona norte de la península y a algunas islas subantárticas (Ochyra et al. 2008). Por otro lado, llama la atención el hallazgo del musgo Bryum orbiculatifolium, especie que antes se ha registrado en pocos sitios. Existen antecedentes previos en cuatro islas subantárticas, en Isla Rey Jorge (Câmara et al. 2017), en Isla Nelson y en Isla Elefante (Pereira \& Putzke 2013), en Tierra de la Reina Maud (Ochyra \& Singh 2008), y sólo en dos sitios en la Isla Decepción (Smith 2005; Ochyra et al. 2008, Pertierra et al. 2017). Esta especie es de especial interés porque ha sido catalogada como muy rara en ecosistemas antárticos (Ochyra et al. 2008, Pertierra et al. 2017), y sus registros en la Isla Decepción estaban restringidos a zonas de actividad geotérmica, los cuales a su vez corresponden a zonas ZAEP (Zona Antártica Especialmente Protegida) (Smith 2005; Pertierra et al. 2017). El hallazgo realizado en este trabajo amplía el registro para esta especie, añadiendo un nuevo sitio en la Isla Decepción, el cual no corresponde a una zona protegida. La especie fue recolectada en el sitio más cercano a la pingüinera. Si bien el sustrato de este sitio concuerda con el descrito por Ochyra et al. (2008) para esta especie, llama la atención la ausencia de actividad geotérmica en este lugar, a diferencia de los registros citados para la especie en la isla. Es importante destacar también que $B$. orbiculatifolium es una especie poco conocida, y probablemente no ha sido recolectada en todos los sitios donde eventualmente habitaría dentro de su amplio rango geográfico (Ochyra et al. 2008). Además, al igual que muchas otras especies de musgos, es probable que sea altamente vulnerable al pisoteo humano (Pertierra et al. 2013), por lo que conocer acerca de su presencia fuera de las zonas ZAEP, y alejada de las zonas de principal actividad geotérmica en la Isla Decepción, permitiría obtener nueva información de esta especie, lo que favorecería su conservación. En este sentido, el resto de las especies detectadas en el transecto ( $y$ en la isla en general) no quedan ajenas al peligro de la actividad humana: la Isla Decepción es una de las más visitadas por turistas. Cada año, desde finales de los 90 , más de 10.000 turistas desembarcan en la costa interior, principalmente en Whalers Bay, Telefon Bay, Baily Head y Pendulum Cove, alcanzando un peak de más de 25.000 turistas de visita en la isla durante los veranos 2006-2007 y 2007-2008 (Pertierra et al. 2014). Así mismo, las dos estaciones científicas aún operativas en la isla, han mantenido un flujo de investigadores que ha ido en aumento en los últimos años, siendo más de 50 personas las que han hecho presencia en la base Gabriel de Castilla durante la época de verano, para desarrollar actividades de investigación (Pertierra et al. 2014). Este flujo, sumado a las frecuentes erupciones volcánicas, mantienen a la isla en un constante estrés sobre la biota que la habita, por lo que profundizar el conocimiento sobre su biodiversidad y la distribución, es imperativo para desarrollar medidas de 
conservación que permitan preservar este ecosistema de la forma más prístina posible.

\section{CONCLUSIONES}

Nuestro estudio muestra diferencias importantes entre las distintas comunidades de musgos asociados a zonas no geotérmicas, e influenciadas por la pingüinera de Punta La Descubierta. Principalmente, la riqueza y cobertura de musgos se ve gradualmente disminuida desde zonas relativamente cercanas a la pingüinera, hacia zonas de baja influencia, existiendo también una variación composicional entre los tres sitios, donde hay 5 especies de musgos comunes a los tres sitios. Cabe destacar que la composición de especies en el sector de Punta La Descubierta, incluye 4 musgos y una hepática que solo están presentes en ese sector y no en los otros dos, alejados de la pingüinera. Por otro lado, la notoria dominancia de $S$. uncinata coincide con el estado temprano en la formación de suelo de la isla, lo que permitiría realizar estudios sobre el desarrollo y formación de éste, entendiendo la Isla Decepción como una zona que presenta estados tempranos en la sucesión ecológica del bioma de tundra polar. Se amplía la cantidad de sitios conocidos para B. orbiculatifolium, añadiendo el primer registro fuera de una zona de actividad geotérmica en la Isla Decepción, en este caso asociado a una pingüinera.

\section{AGRADECIMIENTOS}

Al Instituto Antártico Chileno (INACH, proyecto FR-0418) por el apoyo logístico y a la dotación del ejército de Tierra de la Base Antártica Gabriel de Castilla, quienes brindaron apoyo durante el desarrollo de este trabajo. Se agradece el financiamiento al proyecto FONDECYT 1181745.

\section{REFERENCIAS}

Bockheim, J. 2015. The Soils of Antarctica. World Soils Book Series. 322 pp.

Bokhorst, S., Convey, P., Aerst, R. 2019. Nitrogen Inputs by Marine Vertebrates Drive Abundance and Richness in Antarctic Terrestrial Ecosystems. Current Biology 29(10): 1721-1727.

Câmara, P., Silva, B., Carvalho, M.S., Henriques, D. 2017. The moss flora of Ostrov Geologov (Geologists Island), Maxwell Bay, King George Island, Antarctica. Boletín de la Sociedad Argentina de Botánica 52(2): 251-255.
Casanova-Katny, A., Cavieres, L. 2012 Antarctic Moss carpets facilitate growth of Deschampsia antarctica but not it survival. Polar Biology 35: 1869-1878.

Convey, P. 2005. Antarctic terrestrial ecosystems: responses to environmental change. Polarforschung 75(2-3): 101-111.

Convey, P., Biersma, E.M., Casanova-Katny, A., Maturana, C.S. 2020. Refuges of Antarctic Diversity, In: Oliva, M., RuizFernández, J. (eds.), Past Antarctica. Elsevier, pp. 181-200.

Convey, P., Chown, S.L., Clarke, A., Barnes, D.K.A., Bokhorst, S., Cummings, V., Ducklow, H.W., Frati, F., Green, T.G.A., Gordon, S., Griffiths, H.J., Howard-Williams, C., Huiskes, A.H.L., Laybourn-Parry, J., Lyons, W.B., Mcminn, A., Morley, S.A., Peck, L.S., Quesada, A., Robinson, S.A., Schiaparelli, S., Wall, D.H. 2014. The spatial structure of Antarctic biodiversity. Ecological Monographs 84(2): 203244.

De Pablo, M.A., Molina, A., Recio, C., Ramos, M., Goyanes, G., Ropero, M.A. 2017. Análisis del estado de la capa activa en el emplazamiento de la base antártica española Gabriel de Castilla, Isla Decepción, Antártida. Boletín Geológico y Minero 128(1): 69-92.

Goyanes, G., Vieira, G., Caselli, A., Mora, C., Ramos, M., de Pablo, M.A., Neves, M., Santos, F., Bernardo, I., Gilichinsky, D., Abramov, A., Batista, V., Melo, R., Trindade Nieuwendam, A., Ferreira, A., Oliva, M. 2014. Regimen termico y variabilidad espacial de la capa activa en isla Decepcion, Antartida. Revista de la Asociación Geológica Argentina 71(1): 112-124.

López-Martínez, J., Serrano, E., Schmid, T., Mink, S., Linés, C. 2012. Periglacial processes and landforms in the South Shetland Islands (northern Antarctic Peninsula region). Geomorphology 155-156: 62-79.

Ochyra, R., Lewis-Smith, R., Bednaker-Ochyra H. 2008. The Illustrated Moss Flora of Antarctica. Candbridge University Press. Cambridge. 704 pp.

Ochyra, R., Singh, S.M. 2008. Three remarkable moss records from Dronning Maud Land, continental Antarctica. Nova Hedwigia 86(3): 497-506.

Otero, X., De La Peña-Lastra, S., Pérez-Alberti, A., Ferreira, T., Huerta-Diaz, M. 2018. Seabird colonies as important global drivers in the nitrogen and phosphorus cycles. Nature Communications 9(246).

$\varnothing v$ steda, D.O., Lewis Smith, R.I. 2001. Lichens of Antarctica and South Georgia: a guide to their identification and ecology. Cambridge University Press, Cambridge. 424 pp.

Pàllas, R., Smellie, J.L., Casas, J.M., Calvet, J. 2001. Using tephrochronology to date temperate ice: correlation between ice tephras on Livingston Island and eruptive units on Deception Island volcano (South Shetland Islands, Antarctica). Holocene 11(2): 149-160. 
Pereira, A.B., Putzke, J. 2013. The Brazilian research contribution to knowledge of the plant communities from Antarctic ice free areas. Anais da Academia Brasileira de Ciências 85(3): 923-935.

Pertierra, L., Lara, F., Tejedo, P., Quesada, A., Benayas, J. 2013. Rapid denudation processes in cryptogamic communities from Maritime Antarctica subjected to human trampling. Antarctic Science 25(2): 318-328.

Pertierra, L.R., Tejedo, P., Benayas, J. 2014. Historical Developments, Drivers of Change and Future Scenarios for Human Activities on Deception Island. En: Tin, T., Liggett, D., Maher, P., Lamers, M. (eds.), Antarctic Futures. Springer, Dordrecht.

Pertierra, L.R., Lara, F., Benayas, J., Lewis-Smith, R.I., Hughes, K.A. 2017. Conflicting science requirements impact on rare moss conservation measures. Antarctic Science 30(1): 13-21.

Ramos, M., Vieira, G., De Pablo, M.A., Molina, A., Abramov, A., Goyanes, G., 2017. Recent shallowing of the thaw depth at Crater Lake, Deception Island, Antarctica (2006-2014). Catena 149: 519-528.

Robinson, S., King, D., Bramley-Alves, J., Waterman, M., Ashcroft, M., Wasley, J., Turnbull, J., Miller, R., Ryan-Colton, E., Benny, T., Mulany, K., Clarke, L., Barry, L., Hua, Q. 2018. Rapid change in East Antarctic terrestrial vegetation in response to regional drying. Nature Climate Change 8(10): 879-884.

Simonov, I.M. 1977. Physical geographic description of Fildes Peninsula (South Shetland Islands). Polar Geography 1(3): 223-242.

Skinner, R.A., Ineson, P., Jones, H., Sleep, D., Rank, R. 2006. Using $\delta 15 \mathrm{~N}$ values to characterise the nitrogen nutrient pathways from intensive animal units. Rapid Communications in Mass Spectrometry 20(19): 28582864.

Smellie, J.L. 2002. Geology. En: Geology and geomorphology of Deception Island, pp. 11-30. British Antarctic Survey BAS GEOMAP Series, Cambridge.

Smith, R.I.L. 2005. The thermophilic bryoflora of Deception Island: unique plant communities as a criterion for designating an Antarctic Specially Protected Area. Antarctic Science 17(1): 17-27

Smykla, J., Wołek, J., Barcikowski, A. 2007. Zonation of Vegetation Related to Penguin Rookeries on King George Island, Maritime Antarctic. Arctic, Antarctic, and Alpine Research 39(1): 143-151.

Sørensen, T. 1957. A method of establishing groups of equal amplitude in plant sociology based on similarity of species and its application to analyses of the vegetation on Danish commons. Kongelige Danske Videnskabernes Selskab 5(4): 1-34.

Tatur, A., Myrcha, A. 1989. Soils and vegetation in abandoned penguin rookeries (maritime antarctic) Proc. NIPR Symp. Polar Biology 2: 181-189.

Tatur, A. 2002. Ornithogenic Ecosystems in the Maritime Antarctic-Formation, Development and Desintegration. En: Geoecology of Antarctic Ice-free Coastal Landscapes (eds.), Beyer, L., Bölter, M., pp. 161-186. Springer, Heidelberg, Germany.

Victoria, F., Albuquerque, M., Pereira, A., Simas, F., Spielmann, A., Schaefer, C. 2013. Characterization and mapping of plant communities at Hennequin Point, King George Island, Antarctica. Polar Research 32(1): 19261.

Received: 07.07.2020

Accepted: 18.12 .2020 